\title{
Exposure and Neuronal Excitation by Wireless Power Transfer for Auricular Vagus Nerve Stimulation
}

\author{
T. Van de Steene, E. Tanghe, L. Martens, W. Joseph \\ Department of Information Technology \\ Ghent University/IMEC \\ Ghent, Belgium \\ tom.vandesteene@ugent.be \\ J. C. Széles \\ Department of Surgery \\ Medical University Vienna \\ Vienna, Austria
}

\author{
S. Kampusch, B. Dabiri Razlighi, E. Kaniusas \\ Institute of Electrodynamics, Microwave and Circuit Engineering \\ Vienna University of Technology \\ Vienna, Austria
}

\begin{abstract}
Inductive wireless power transfer (WPT) can be used to power implanted as well as wearable medical devices, such as a percutaneous auricular vagus nerve stimulation device. This device is placed on the neck of the patient and is connected to needle electrodes in the auricle. With regard to WPT, limitations on exposure to electric and magnetic fields should not be exceeded. Furthermore, these fields should not interfere with the therapeutic goal of stimulation, i.e., with unintended peripheral nerve stimulation in the auricle. These effects are investigated by numerical simulation of induced internal fields in the head and neck and, for the first time, subsequent neuronal simulations, quantifying the potential of neuronal excitation by the fields in the auricle in particular. Internal electric field values were in the range of $1 \%-5 \%$ of the ICNIRP 2010 basic restrictions, and current densities were in the range of $30 \%-45 \%$ of the ICNIRP 1998 basic restrictions, indicating that all tested configurations are conform the guidelines. Basic restrictions on heating of tissue turned out not to be of relevance for this application. Thresholds for neuronal stimulation were two orders of magnitude higher than the induced fields, suggesting that there is almost no risk for unintended stimulation.
\end{abstract}

Index Terms-wireless power transfer (WPT), magnetic field exposure, percutaneous auricular vagus nerve stimulation (pVNS), ICNIRP guidelines

\section{INTRODUCTION}

Wearable medical devices are playing an increasingly important role in healthcare today. As with any other electrical device, sufficient power is needed to keep the device running, while delivering and storing this energy might be a challenge. Wireless power transfer (WPT) by means of inductive coupling has a number of advantages that often make it the best suited method for several medical devices.

In particular, WPT can be used to power percutaneous auricular vagus nerve stimulation (pVNS) devices such as in [1] and [2]. These devices are placed on the head or neck as shown in Fig. 1, and are connected to a number of needle electrodes which are placed in the auricle. While this is not an implanted device, WPT offers a number of benefits over a wired connection for power supply. Since no physical connection is needed for power transfer and/or data transfer, the device design can be optimized towards a green technology.

However, a possible side effect to take into account, is the generated magnetic fields. The resulting induced electric fields and circular currents might lead to peripheral nerve stimulation and to a lesser extent, heating, although the latter is not expected to be an important factor at the frequencies of WPT $(100 \mathrm{kHz}-200 \mathrm{kHz})$. Limits on the specific absorption rate (SAR), which are intended to restrict heating in the body, have to be considered at frequencies between $100 \mathrm{kHz}$ and $10 \mathrm{GHz}$ [3]. However, limitations on current densities (which have to be considered in the range $1 \mathrm{~Hz}-10 \mathrm{MHz}$ [3]) are more likely to be critical than limitations on SAR.

Numerical exposure studies have already been conducted for active implantable medical devices and WPT in the proximity of the body in humans and animals [4], [5], [6], [7]. These studies have not yet combined an exposure assessment with subsequent neuronal simulations, directly quantifying the effect on peripheral nerves.

This study aims to quantify the electric and magnetic fields in the head and neck, and more specifically in the auricle by means of numerical simulations using a heterogeneous human head model. Subsequently, these values will be compared to the applicable limitations on exposure of humans to electromagnetic fields as defined by the International Commission on Non-Ionizing Radiation Protection (ICNIRP) [3], [8]. Finally, the actual potential of these fields to stimulate neurons in the auricle will be investigated. This is particularly interesting in the case of pVNS due to the proximity of the target neurons in the auricle to the coils used for WPT. Of course it should 
be avoided that WPT has an influence on the fields in the auricle in such a way that the intended stimulation by needle electrodes is disturbed with potentially altered therapeutic effect as a result.

Moreover, it is interesting to compare the ICNIRP basic restrictions (BR), which are meant among others to limit peripheral nerve stimulation, with actual stimulation thresholds for the case of WPT for $\mathrm{pVNS}$.

\section{MODELS AND METHODS}

The electric and magnetic fields, exposure and neural activation resulting from WPT to transfer energy to a wearable pVNS device are investigated [9]. Numerical models of both the pVNS device and a human head are used in combination with simulation software to obtain the internal fields.

\section{A. Model of $p V N S$ device}

The model of the pVNS device, wherein WPT takes place, consists of a plastic housing, a transmitter coil with 10 turns attached to a $0.5 \mathrm{~mm}$ circular ferrite plate and a receiver coil with two layers of 11 turns each, a battery with metal housing, and fiberglass PCBs.

The magnetic field of the coils is excited by a source current in the primary coil which is limited to $2 \mathrm{~A}$. Simulations are carried out at three different frequencies: $130 \mathrm{kHz}$, which is the intended frequency of use, $100 \mathrm{kHz}$ and $200 \mathrm{kHz}$, which define a usual range for WPT applications, according to [10].

\section{B. Anatomical models}

For the human head, the MIDA-model [11] was used, which is a high resolution model containing 153 structures of the head. This model is used to assess the exposure and maximum field values in the different tissues, which are to be compared to the exposure guidelines [3], [8]. Values of electrical and magnetic properties of the different tissues are included in the model. Only the conductivity of the skin was changed. This value was set to $0.2 \mathrm{~S} / \mathrm{m}$, according to [12] instead of $0.00045 \mathrm{~S} / \mathrm{m}(100 \mathrm{kHz}), 0.0006 \mathrm{~S} / \mathrm{m}(130 \mathrm{kHz})$ and $0.001 \mathrm{~S} / \mathrm{m}(200 \mathrm{kHz})$ as defined by the original model [11]. In order to be able to investigate the effect of the fields in the auricle with sufficient detail, a high resolution and more realistic model of the auricle by [2], especially composed for the investigation of pVNS, was added to the simulations. This anatomical model contains neurons corresponding to the locations of the auricular branch of the vagus nerve. Both the auricular cartilage and skin have conductivity values of $0.2 \mathrm{~S} / \mathrm{m}$ [2]. The model and locations of neurons is illustrated in Fig. 2.

For the reason of possible variations in the placement of the device, four different configurations are defined. In all four cases, the device is placed behind and slightly below the ear, but small variations in position and rotation angle exist. These configurations are called $\mathrm{C} 1-\mathrm{C} 4$, and are shown in Fig 1. For $\mathrm{C} 4$ the auricle is closer to the coils than practical with respect to the housing dimensions, and could therefore be considered as a worst-case scenario.

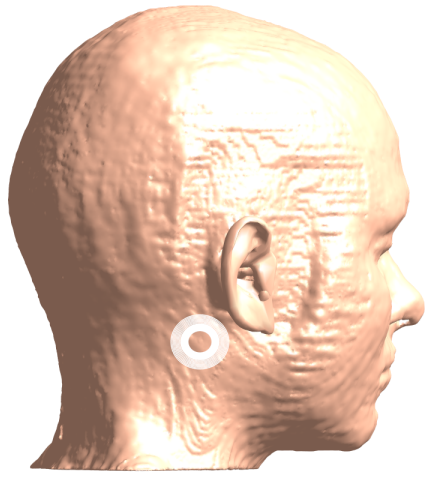

(a)

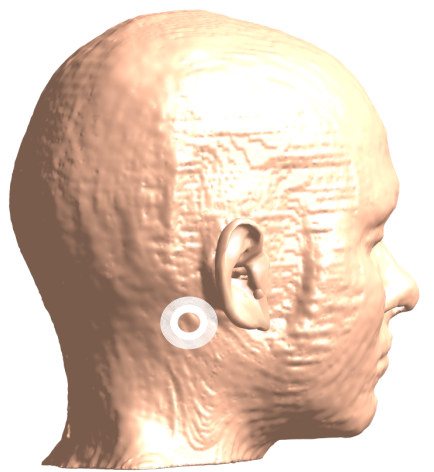

(c)

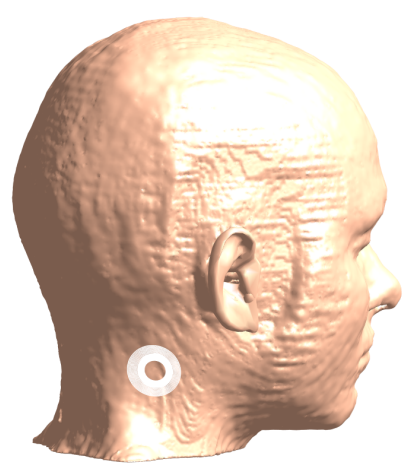

(b)

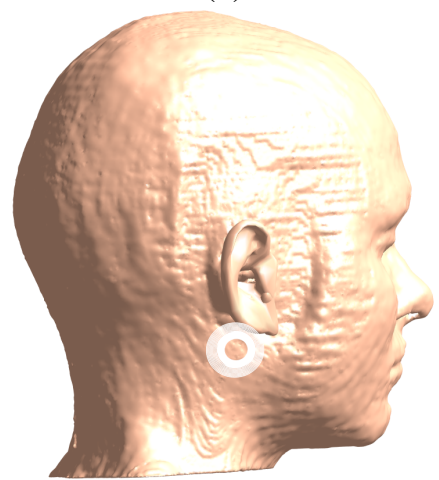

(d)
Fig. 1. (a) - (d) Placement of the essential components for WPT (white) in the pVNS device behind the auricle for the different configurations $\mathrm{C} 1-\mathrm{C} 4$ respectively.

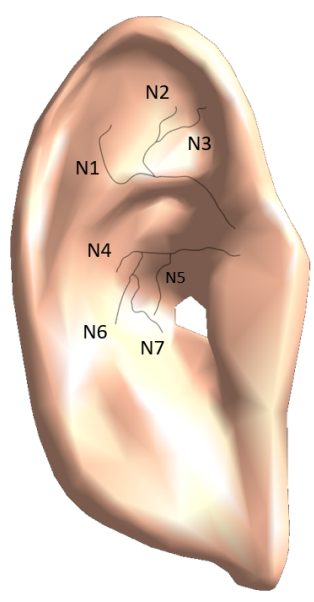

Fig. 2. Detailed view of the $3 \mathrm{D}$ model of the auricle and neuron locations for neuronal simulations [2]. 


\section{Electromagnetic simulations}

Simulations are carried out using Sim4Life [13], according to the numerical methodology of a similar study by [7]. The quasi-static electromagnetic approximation was used. The first step is to calculate the magnetic fields arising from the currents in transmitting and receiving coils. For this case, the Magnetic Vector Potential solver of the software is used, which calculates the $\mathrm{H}$ field, the B field and magnetic vector potential A for a given source current. The computational domain can consist of materials with variable magnetic permeability, perfect magnetic conductors and perfect electric conductors. The transmitting coil was operated with the maximum current of $2 \mathrm{~A}$. The receiving coil is modeled as a perfect electrical conductor.

Secondly, the Magneto Quasi-Static solver is used to calculate the electric field and current density field in the lossy domain $(\sigma \neq 0)$, assuming that ohmic currents dominate displacement currents. From these fields, the quantities of interest for the ICNIRP BRs [3], [8] can be automatically calculated: For the electric field, the 99\% value is taken for each tissue while current densities are averaged over a $1 \mathrm{~cm}^{2}$ circular area perpendicular to the direction of the current. The SAR is calculated as well, averaging over $10 \mathrm{~g}$ of tissue.

\section{Neuronal simulations}

For the neuronal simulations, the internal fields in the anatomical model of the human auricle of [2] are used as an input. This model contains seven axons, located at regions of the auricle where the vagus nerve is prominent. The used neuronal model is the spatially extended non-linear node model (SENN-model) [14]. In order to simulate the worst case scenario, the axon diameter was set to $12 \mu \mathrm{m}$, which is the largest of possible diameters of the neurons of the vagus nerve in the auricle [15] and are therefore the most easily excitable [16]. The titration factor, which is the factor by which the stimulus (in this case the fields generated during WPT) has to be multiplied in order to induce spiking of axons, is calculated by the software. A titration factor larger than unity means that neurons will not be stimulated during WPT.

\section{RESULTS AND DISCUSSION}

\section{A. Electric and current density field distributions}

From the simulations for each of the configurations C1-C4 and each of the investigated frequencies $(100 \mathrm{kHz}-130 \mathrm{kHz}$ - $200 \mathrm{kHz}$ ), the electric field and current density field in the head and neck were obtained. Fig. 3 (a) and Fig. 3 (b) show the resulting fields for configuration $\mathrm{C} 1$ at $100 \mathrm{kHz}$.

The highest values for the electric field as well as for the current density are found in the skin of the ear and/or close to the device, depending on the configuration. The influence of varying tissue conductivity is visible as well, especially for the current density field. The $99 \%$ electric field value was extracted for each individual tissue and surfaceaveraged current density values were calculated for the whole model. Maximum values are shown in Table I and Table II respectively, together with the ICNIRP BR.

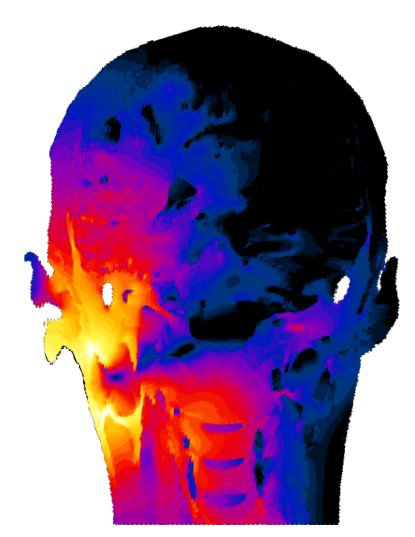

(a)

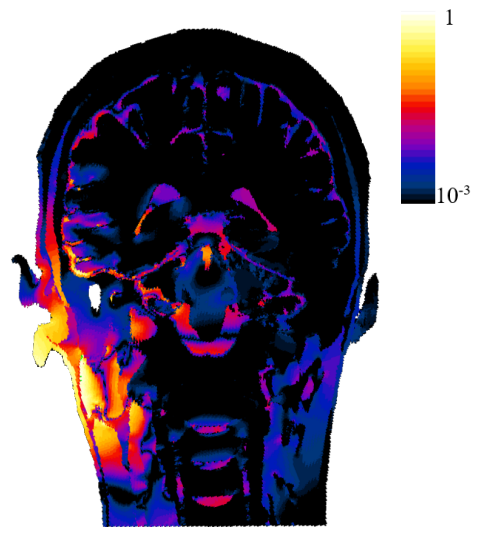

(b)
Fig. 3. (a) Electric field normalized to $0.7 \mathrm{~V} / \mathrm{m}$ and (b) current density field normalized to $0.1 \mathrm{~A} / \mathrm{m}^{2}$ in a coronal slice of the head through the ear.

Higher values are found at higher frequencies, which is in accordance with Faraday's law of induction. When comparing different configurations, it can be seen that the maximum electric field values can differ by a factor three. Configuration $\mathrm{C} 2$ results in the lowest and $\mathrm{C} 4$ in the highest values for every frequency.

\section{B. Compliance with guidelines}

From the results in section III-A, it is clear that the relevant maximum values for both the electric field and current density field are below the ICNIRP BR. This is visualized in Fig. 4 and Fig. 5, which show the maximum field values as a percentage of the ICNIRP BR for each frequency. The values of the electric field are between $1 \%$ and $5 \%$ of the BR. This means that it is highly unlikely that the limits will be exceeded due to WPT by the device. For the current density, values are between

TABLE I

99\% ELECTRIC FIELD VALUES FOR VARYING FREQUENCY AND CONFIGURATION $(\mathrm{V} / \mathrm{m})$

\begin{tabular}{|c|c|c|c|}
\hline \multirow{2}{*}{ Configuration } & \multicolumn{3}{|c|}{ Frequency } \\
\cline { 2 - 4 } & $\mathbf{1 0 0} \mathbf{~ k H z}$ & $\mathbf{1 3 0} \mathbf{~ k H z}$ & $\mathbf{2 0 0} \mathbf{~ k H z}$ \\
\hline C1 & 0.30 & 0.39 & 0.61 \\
\hline C2 & 0.21 & 0.27 & 0.42 \\
\hline C3 & 0.34 & 0.44 & 0.68 \\
\hline C4 & 0.59 & 0.76 & 1.17 \\
\hline ICNIRP BR & 13.50 & 17.55 & 27.00 \\
\hline
\end{tabular}

TABLE II

MAXIMUM SURFACE-AVERAGED CURRENT DENSITY FIELD VALUES FOR VARYING FREQUENCY AND CONFIGURATION $\left(\mathrm{A} / \mathrm{m}^{2}\right)$

\begin{tabular}{|c|c|c|c|}
\hline \multirow{2}{*}{ Configuration } & \multicolumn{3}{|c|}{ Frequency } \\
\cline { 2 - 4 } & $\mathbf{1 0 0} \mathbf{~ k H z}$ & $\mathbf{1 3 0} \mathbf{~ k H z}$ & $\mathbf{2 0 0} \mathbf{~ k H z}$ \\
\hline C1 & 0.08 & 0.10 & 0.15 \\
\hline C2 & 0.06 & 0.08 & 0.12 \\
\hline C3 & 0.07 & 0.09 & 0.13 \\
\hline C4 & 0.09 & 0.11 & 0.18 \\
\hline ICNIRP BR & 0.20 & 0.26 & 0.40 \\
\hline
\end{tabular}




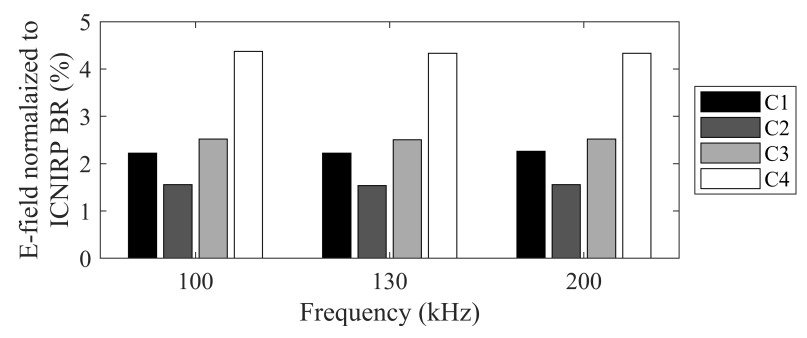

Fig. 4. 99\% Electric field values for different configurations and frequencies normalized to the ICNIRP BR.

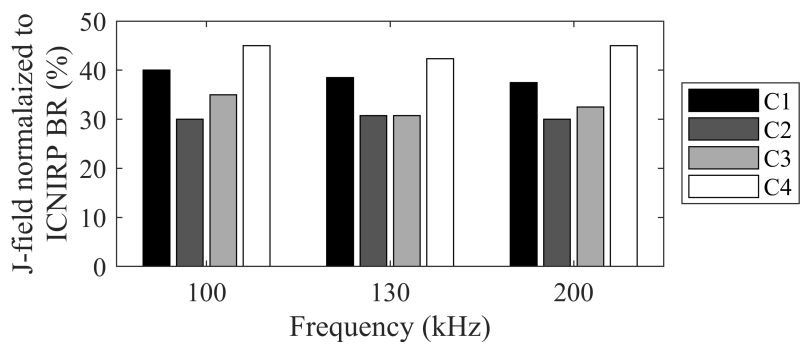

Fig. 5. Maximum current density values for different configurations and frequencies normalized to the ICNIRP BR.

$30 \%$ and $45 \%$ of the BR. While this is considerably higher than for the electric field, there is still more than a factor two of margin. It is also seen that variation of maximum current densities is lower than for electric field values (III-A). This lower variation suggests that other configurations than the four configurations tested, are less likely to induce current densities exceeding the limits, although only additional simulations can confirm this presumption.

The SAR was calculated as well, and all values were between $0.00021 \%(\mathrm{C} 2$ at $100 \mathrm{kHz})$ and $0.00168 \%(\mathrm{C} 4$ at $200 \mathrm{kHz}$ ) of the $2 \mathrm{~W} / \mathrm{kg} \mathrm{BR}$ [3]. This was as expected at the relatively low investigated frequencies.

\section{Neuronal excitation thresholds}

The electric fields in the auricle were used to simulate the response of seven neurons by calculation of the titration factor. The results are summarized in Fig. 6. For each combination of configuration number and frequency, the minimum value from all seven neurons was taken.

Fig. 6 shows that the titration factors are very high. This indicates that the needed electric field for stimulation of any of the neurons needs to be more than two orders of magnitude higher than the actual field. It is very unlikely that WPT will result in neuronal stimulation and therefore interfere with the therapeutic goal of the device. These results are expected since the field values are below the ICNIRP BR. The lowest titration factors were obtained for neurons N6 and N7 (276 and 342 respectively). These are the closest to the device and correspond with the locations of high electric fields as well.

While these results show that no neural stimulation will be seen, there is still a large variation of the results (a factor 36 between $\mathrm{N} 3$ and N6) for only a limited total number of

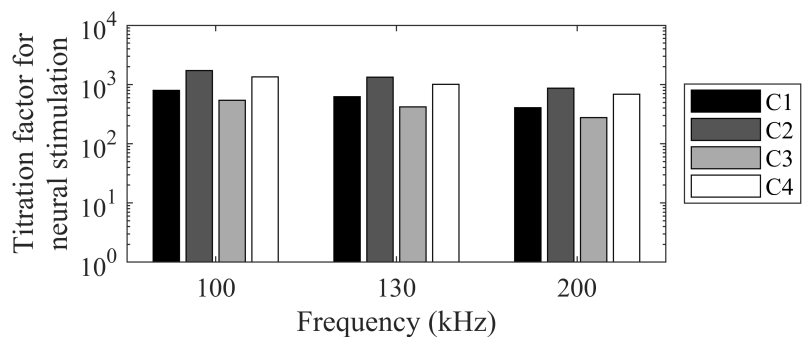

Fig. 6. Minimum values of the titration factor for varying frequency and configuration, indicating that the investigated axons will not be stimulated as a result of WPT.

neurons. Therefore, investigation of more neurons with varying geometries and locations on the auricle, might add to the statistical significance of the results.

\section{CONCLUSION}

The exposure of patients to the induced electric fields and current density fields by WPT was investigated by numerical simulations at different frequencies and different configurations, covering inter-user variability. The electric fields were in the range of $1 \%-5 \%$ of the ICNIRP BR and the current density fields were in the range of $30 \%-45 \%$, indicating that the device is conform the guidelines for the tested setups. SAR values, which are four orders of magnitude or more below the limits, turned out to be irrelevant for this application at the investigated frequencies.

For the first time, the generated fields by WPT were directly used for neuronal simulations, which is especially interesting for pVNS, due to the proximity of the WPT device and the stimulation region. These simulations indicated that there is almost no risk for interference between WPT and pVNS, since stimulation thresholds were two orders of magnitude or more higher than the induced fields.

Seeing the variation between different configurations, investigation of additional configurations and non-sinusoidal waveforms/harmonics might be needed to confirm these results. Comparison of numerical simulations to measured field values around the device would add to the validity of the results as well.

\section{ACKNOWLEDGMENT}

This work was supported by the Research Foundation Flanders (FWO-V) under grant agreement No G003415N.

\section{REFERENCES}

[1] S. Kampusch, E. Kaniusas, F. Thürk, D. Felten, I. Hofmann, and J. C. Széles, "Device development guided by user satisfaction survey on auricular vagus nerve stimulation," Current Directions in Biomedical Engineering, vol. 2, no. 1, jan 2016. [Online]. Available: https://www.degruyter.com/view/j/cdbme.2016.2.issue1/cdbme-2016-0131/cdbme-2016-0131.xml

[2] A. M. Samoudi, S. Kampusch, E. Tanghe, J. C. Széles, L. Martens, E. Kaniusas, and W. Joseph, "Numerical modeling of percutaneous auricular vagus nerve stimulation: a realistic 3D model to evaluate sensitivity of neural activation to electrode position," Medical and Biological Engineering and Computing, 2017. 
[3] International Commission on Non-Ionizing Radiation Protection, "Guidelines for limiting exposure to time-varying electric, magnetic, and electromagnetic fields (up to $300 \mathrm{GHz}$ )," Health physics, vol. 74, no. 4, pp. 494-522, apr 1998. [Online]. Available: http://www.ncbi.nlm.nih.gov/pubmed/9525427

[4] T. Campi, S. Cruciani, F. Palandrani, V. De Santis, A. Hirata, and M. Feliziani, "Wireless power transfer charging system for AIMDs and pacemakers," IEEE Transactions on Microwave Theory and Techniques, 2016.

[5] X. L. Chen, A. E. Umenei, D. W. Baarman, N. Chavannes, V. D. Santis, J. R. Mosig, and N. Kuster, "Human Exposure to Close-Range Resonant Wireless Power Transfer Systems as a Function of Design Parameters," IEEE Transactions on Electromagnetic Compatibility, vol. 56, no. 5, pp. 1027-1034, oct 2014. [Online]. Available: http://ieeexplore.ieee.org/document/6766202/

[6] F. Wen and X. Huang, "Human Exposure to Electromagnetic Fields from Parallel Wireless Power Transfer Systems." International journal of environmental research and public health, vol. 14, no. 2, 2017. [Online]. Available: http://www.ncbi.nlm.nih.gov/pubmed/28208709 http://www.pubmedcentral.nih.gov/articlerender.fcgi?artid=PMC533471

[7] S. Benaissa, A. M. Samoudi, D. Plets, G. Vermeeren, L. Verloock, B. Minnaert, N. Stevens, L. Martens, F. A. Tuyttens, B. Sonck, and W. Joseph, "Numerical assessment of EMF exposure of a cow to a wireless power transfer system for dairy cattle," Computers and Electronics in Agriculture, vol. 151, pp. 219-225, aug 2018. [Online]. Available: https://linkinghub.elsevier.com/retrieve/pii/S0168169917314898

[8] International Commission on Non-Ionizing Radiation Protection, "Guidelines for limiting exposure to time-varying electric and magnetic fields $(1 \mathrm{~Hz}-100 \mathrm{kHz}), "$ Health physics, vol. 99, no. 6, pp. 818-836, 2010. [Online]. Available: http://www.icnirp.org/cms/upload/publications/ICNIRPLFgdl.pdf

[9] "SzeleSTIM," 2018. [Online]. Available: www.szelestim.com

[10] Wireless Power Consortium, "System description wireless power transfer," Tech. Rep. October, 2010.

[11] M. I. Iacono, E. Neufeld, E. Akinnagbe, K. Bower, J. Wolf, I. Vogiatzis Oikonomidis, D. Sharma, B. Lloyd, B. J. Wilm, M. Wyss, K. P. Pruessmann, A. Jakab, N. Makris, E. D. Cohen, N. Kuster, W. Kainz, and L. M. Angelone, "MIDA: A Multimodal ImagingBased Detailed Anatomical Model of the Human Head and Neck," PLOS ONE, vol. 10, no. 4, pp. 1-35, 2015. [Online]. Available: https://doi.org/10.1371/journal.pone.0124126

[12] V. De Santis, X. L. Chen, I. Laakso, and A. Hirata, "An equivalent skin conductivity model for low-frequency magnetic field dosimetry," Biomedical Physics \& Engineering Express, 2015.

[13] Zurich MedTech, "Sim4Life," 2018. [Online]. Available: zmt.swiss/sim4life/

[14] J. P. Reilly, V. T. Freeman, and W. D. Larkin, "Sensory effects of transient electrical stimulation-evaluation with a neuroelectric model," IEEE Trans Biomed Eng, vol. 32, no. 12, pp. 1001-1011, dec 1985.

[15] S. Safi, J. Ellrich, and W. Neuhuber, "Myelinated Axons in the Auricular Branch of the Human Vagus Nerve," Anatomical Record, vol. 299, no. 9 , pp. 1184-1191, 2016.

[16] A. M. Samoudi, "Electromagnetic Modelling and Optimization for SPECT-MRI and Auricular Vagus Nerve Stimulation," Ph.D. dissertation, Ghent University, 2017. [Online]. Available: https://biblio.ugent.be/publication/8560652/file/8560653 